\title{
Expression and clinical significance of estrogen-regulated long non-coding RNAs in estrogen receptor $\alpha$-positive ovarian cancer progression
}

\author{
JUN-JUN QIU ${ }^{1-3}$, LE-CHI YE ${ }^{4}$, JING-XIN DING $^{1-3}$, WEI-WEI FENG ${ }^{1-3}$, \\ HONG-YAN JIN ${ }^{1-3}$, YING ZHANG ${ }^{1-3}$, QING LI ${ }^{1-3}$ and KE-QIN HUA ${ }^{1-3}$ \\ ${ }^{1}$ Department of Gynecology, Obstetrics and Gynecology Hospital, Fudan University, Shanghai 200011; \\ ${ }^{2}$ Department of Obstetrics and Gynecology of Shanghai Medical College, Fudan University, Shanghai 200032; \\ ${ }^{3}$ Shanghai Key Laboratory of Female Reproductive Endocrine Related Diseases, Shanghai 200011; \\ ${ }^{4}$ Department of General Surgery, Zhongshan Hospital of Fudan University, Shanghai 200032, P.R. China
}

Received November 30, 2013; Accepted January 4, 2014

DOI: 10.3892/or.2014.3000

\begin{abstract}
Estrogen (E2) has long been implicated in epithelial ovarian cancer (EOC) progression. The effects of E2 on cancer progression can be mediated by numerous target genes, including coding RNAs and, more recently, non-coding RNAs (ncRNAs). Among the ncRNAs, long ncRNAs (lncRNAs) have emerged as new regulators in cancer progression; therefore, our aim was to determine whether the expression of any lncRNAs is regulated by $\mathrm{E} 2$ and, if so, whether a subset of these lncRNAs have some clinical significance in EOC progression. A microarray was performed to identify E2-regulated lncRNAs in E2 receptor (ER) $\alpha$-positive EOC cells. Bioinformatics analyses of lncRNAs were conducted, focusing on Gene Ontology and pathway analyses. Quantitative real-time polymerase chain reactions were performed to confirm the expression of certain IncRNAs in ER $\alpha$-positive EOC tissues. The correlation between certain IncRNA expression and clinicopathological factors as well as prognosis in ER $\alpha$-positive EOC patients was then analyzed. We showed that 115 lncRNAs exhibited significant changes in E2-treated SKOV3 cells compared with untreated controls. Most of these IncRNAs were predicated to have potential to contribute to cancer progression. Notably, three candidates (TC0100223, TC0101686 and TC0101441) were aberrantly expressed in ER $\alpha$-positive compared to ER $\alpha$-negative EOC tissues, showing correlations with some malignant cancer phenotypes such as advanced FIGO stage and/or high histological grade. Furthermore, multivariate analysis indicated that TC0101441 was an independent prog-
\end{abstract}

Correspondence to: Professor Ke-Qin Hua, Department of Gynecology, Obstetrics and Gynecology Hospital, Fudan University, 419 Fangxie Road, Shanghai 200011, P.R. China

E-mail: huakeqin@126.com

Key words: estrogen, lncRNA, ovarian cancer, prognosis nostic factor for overall survival. Taken together, these results indicate for the first time that E2 can modulate lncRNA expression in ER $\alpha$-positive EOC cells and that certain lncRNAs are correlated with advanced cancer progression and suggestive of a prognostic indicator in ER $\alpha$-positive EOC patients. Knowledge of these E2-regulated lncRNAs could aid in the future understanding of the estrogenic effect on EOC progression and may assist in the clinical design of new target therapies based on a perspective of lncRNA.

\section{Introduction}

Epithelial ovarian cancer (EOC) is the most deadly malignancy of the female reproductive tract in many countries $(1,2)$. Involvement of steroid hormones, primarily estrogen, has been associated with EOC. Ample evidence from epidemiologic, clinical and experimental research has demonstrated that E2 is responsible for promoting EOC progression (3-9). Although the effects of E2 on EOC progression have been extensively studied, the underlying mechanisms remain unknown and the clinical response to steroid hormone therapy remains disappointing. Thus, fully identifying the contributions of E2 to EOC progression is urgently required.

Compelling data have demonstrated that the effects of E2 on EOC development are mediated by the regulation of target genes involved in the control of cancer progression. Previous studies, including ours, have identified a panel of aberrantly expressed E2-regulated protein-coding genes that are involved in cellular growth control, such as cyclin D1 and c-myc, and in cellular metastasis control, such as fibulin-1, cathepsin-D, HIF-1, nm23-H1, E-cadherin and MMP-2 (4-7,10,11). Despite these protein-coding genes, undoubtedly, the set of genes that directly mediate estrogenic effects on EOC progression has not been fully defined. Therefore, exploration of new E2-regulated genes is needed, which may help elucidate estrogenic effects on EOC progression and provide optional therapeutic targets.

The human transcriptome was found to be more complex than a collection of protein-coding genes, showing extensive non-coding RNA (ncRNA) expression (12). Long ncRNAs 
(lncRNAs; >200 nt in length), initially argued to be spurious transcriptional noise (13), are emerging as new regulators in the cancer paradigm. Aberrant expression of lncRNAs has been reported to be associated with malignant phenotypes in various human tissues, and some lncRNAs, such as HOTAIR, MALAT-1, H19, HULC, lincRNA-p21 and MEG3, might also function as tumor suppressor genes or oncogenes (14-19). Although several published studies have reported lncRNAs such as lncRNA-LSINCT5 and HOST2 in EOC $(20,21)$, to our knowledge, no studies have focused on E2-regulated lncRNAs in EOC.

Therefore, we sought to identify E2-regulated lncRNAs in EOC. We found that E2 stimulation of ER $\alpha$-positive $(E R \alpha+)$ EOC cells resulted in a panel of differentially expressed IncRNAs, showing great potential to contribute to cancer progression based on bioinformatics analyses. Moreover, we found that some candidate lncRNAs were aberrantly expressed in $\mathrm{ER} \alpha+$ compared to ER $\alpha$-negative (ER $\alpha$-) EOC tissues, and their differential expression was associated with certain clinicopathological variables and poor prognosis of ER $\alpha+$ EOC patients. Our results highlight for the first time the potential use of lncRNAs as causal link with estrogenic effects on EOC progression and as surrogate targets to hormone therapy.

\section{Materials and methods}

Cells and treatment. The ovarian cancer cell line SKOV3 was obtained from the American Type Culture Collection (ATCC; Manassas, VA, USA). SKOV3 cells were routinely maintained in RPMI-1640 medium (Gibco-BRL, Gaithersburg, MD, USA) supplemented with $10 \%$ fetal bovine serum (FBS; Gibco) and maintained at $37^{\circ} \mathrm{C}$ with $5 \% \mathrm{CO}_{2}$. For the E2 induction experiments, cells (plated at 20-30\% confluence) were grown for 3 days in phenol red-free RPMI-1640 (Gibco) containing $5 \%$ activated, charcoal-treated foetal bovine serum (Serana, Bunbury, Australia). Next, the cells were treated for $24 \mathrm{~h}$ with $10^{-8}$ M E2 or vehicle alone (DMSO, $0.01 \%$ of final volume) as a control.

RNA extraction and microarray. TRIzol (Invitrogen, Carlsbad, CA, USA) was used to extract total RNA from SKOV3 cells with or without $24-\mathrm{h}$ treatment with $10^{-8} \mathrm{M}$ E2. There were three replicates of each sample; they were purified using an RNeasy Micro kit (cat. \#74004; Qiagen GmbH, Hilden, Germany). An Agilent Bioanalyzer 2100 (Agilent Technologies, Santa Clara, CA, USA) was used to quantify the RNA and evaluate its integrity; the $28 \mathrm{~S}: 18 \mathrm{~S}$ ratio was determined, and an RNA integrity number (RIN) was assigned to each sample. RNA with no evidence of degradation and no signs of DNA contamination (as indicated by an RIN $\geq 7.0$ and a $28 \mathrm{~S}: 18 \mathrm{~S}$ ratio $\geq 0.7$ ) was processed for further analysis.

The IncRNA and mRNA expression profiles were obtained using the Glue Grant Human Transcriptome (GG-H) Array, which was manufactured by Affymetrix and Stanford University. This array contains 5,869 probes covering 730 non-coding, functional RNAs and 3,292,929 probes covering 27,670 coding genes collected from RefSeq, Ensembl and UCSC Known Genes, based on human genome assembly hg18 (22). The array experiments and computational analysis were performed according to the manufacturer's instructions
(Affymetrix, Santa Clara, CA, USA). Briefly, $0.2 \mu \mathrm{g}$ of total RNA was amplified and labelled, and $20 \mu \mathrm{g}$ of labelled cDNA was loaded onto the array. The array was hybridized and washed using the GeneChip ${ }^{\circledR}$ Hybridization, Wash and Stain kit (cat. \#900720), Hybridization Oven 645 (cat. \#00-0331-220V) and Fluidics Station 450 (cat. \#00-0079). The slides were scanned in a GeneChip ${ }^{\circledR}$ Scanner 3000 (cat. \#00-00212). The raw data were obtained using Command Console Software 3.1 with the default settings and were processed using Affymetrix Power Tools with Robust Multiarray Analysis (RMA) for background correction, normalization and summarization. Differentially expressed genes [defined as a fold-change $\geq 1.5$ and a P-value $<0.05$ (t-test)] were selected for further study.

Bioinformatics functional analysis of E2-regulated lncRNAs Identification of lncRNA-mRNA targeting pairs. Two procedures were performed to search for the target mRNAs of lncRNAs. First, UCSC hg18 (http://genome.ucsc.edu/) was used to predict lncRNA targets. Target genes under cis-regulatory control were defined as genes whose transcription was regulated by lncRNAs in nearby genomic locations ( $\leq 10 \mathrm{kbp}$ upstream or downstream) (23). Based on mRNA sequence complementarity and RNA duplex energy prediction, transacting target genes were identified using BLAST software in the first round of screening (with the parameter e $<1 \mathrm{E}-5$ ) and RNAplex software for final verification (with the parameter -e -20) (24). Additionally, to improve the accuracy of the target prediction, the predicted lncRNA targets (both cis and trans) were combined with the differentially expressed mRNAs in the profile. The resultant overlapping mRNAs were considered the final putative targets of the differentially expressed lncRNAs. This information formed the basis for determining the IncRNA-mRNA targeting pairs.

Gene ontology $(G O)$ and pathway analysis. For the GO and pathway analyses, the putative targets were initially inputted into the Database for Annotation, Visualization and Integrated Discovery (DAVID, http://david.abcc.ncifcrf.gov/), which searched the GO terms to identify the molecular function represented in the gene profile (25), and then into the database of the Kyoto Encyclopedia of Genes and Genomes (KEGG; http://www.genome.ad.jp/kegg/) to analyze the roles of the targets in molecular pathways (26).

Tissue samples and patient data. The study included 95 patients who underwent surgery for primary ovarian cancer in the Department of Gynecology, Obstetrics and Gynecology Hospital of Fudan University between January 2006 and December 2008. Patients were included based on the availability of tissue and follow-up data. Patients with borderline ovarian tumors or with two or more different malignancies were excluded from the study. None of the patients had received preoperative radiotherapy, chemotherapy or hormonal therapy. All EOC tissue samples were frozen immediately after surgery and stored in liquid nitrogen until use.

Clinical and pathological data of EOC patients were evaluated by reviewing medical charts and the original pathology reports. Staging and grading were evaluated in accordance with the criteria of the International Federation of Gynecologists and Obstetricians (FIGO) and the World Health Organization 
Table I. Primer sequences of the studied genes.

\begin{tabular}{lll}
\hline Gene & Primer & \multicolumn{1}{c}{ Sequence (5'-3') } \\
\hline TC1500845 & F: & ACCACGACTCCCAAGAGGTA \\
& R: & CAGCTGCGATGGTGAGAACT \\
TC0101441 & F: & CAAGGCAGGTGAGAACGAGT \\
& R: & CTCGACTTAGGGAGCTGCAC \\
TC0100223 & F: & ATGAGGGCTCTGCTCTATGAATGG \\
& R: & GGCTTGTTCAGTGTCTGTTAAGGGT \\
TC0101686 & F: & GGCTACTTACATGGTCCAGCA \\
& R: & TAGCATGGAAAGGACCACTGC \\
GAPDH & F: & TGACTTCAACAGCGACACCCA \\
& R: & CACCCTGTTGCTGTAGCCAAA
\end{tabular}

F, forward; R, reverse.

(WHO). Follow-up data were obtained by reviewing the out patient charts, contacting patients or correspondence. Overall survival (OS) was calculated from the date of surgery until the date of mortality or end of follow-up (January 2013). The present study was approved by the Research Ethics Committee of Fudan University, China. Informed consent was obtained from all the patients.

Immunohistochemistry. The immunohistochemical study of $\mathrm{ER} \alpha$ was performed using a standard streptavidin-peroxidase method. The endogenous peroxidase activity was blocked with $3 \% \mathrm{H}_{2} \mathrm{O}_{2}$ for $10 \mathrm{~min}$. For the antigen retrieval, slides were immersed in $10 \mathrm{mM}$ citrate buffer ( $\mathrm{pH}$ 6.0) and boiled for $15 \mathrm{~min}$ in a microwave oven. Non-specific binding was blocked by $5 \%$ normal goat serum for $10 \mathrm{~min}$. The slides were incubated with a 1:50 dilution of monoclonal antibody against ER $\alpha$ (Santa Cruz Biotechnology, Santa Cruz, CA, USA) at $4^{\circ} \mathrm{C}$ overnight in a moist chamber. The slides were sequentially incubated with biotinylated goat anti-mouse IgG (1:100 dilution; Santa Cruz Biotechnology) and then streptavidin-peroxidase conjugate, each for $30 \mathrm{~min}$ at room temperature. Isotope-matched human IgG was used in each case as a negative control. Finally, the 3,5-diaminobenzidine (DAB) Substrate kit (Dako) was used for color development followed by Mayer's hematoxylin counterstaining. ER $\alpha+$ cases were defined as tumors with $>10 \%$ stained nuclei (27).

Quantitative real-time reverse transcription polymerase chain reaction ( $q R T-P C R)$. qRT-PCR analysis of IncRNA expression was performed using FastStart Universal SYBR-Green Master (Rox; Roche) and an ABI Prism 7900 Real-Time PCR system (Applied Biosystems, Foster City, CA, USA). Briefly, total RNA was extracted from cells and tissues and converted to cDNA using an iScript cDNA Synthesis kit (Bio-Rad Laboratories) according to the manufacturer's protocol. The PCR amplifications were performed in a $10-\mu 1$ (total volume) reaction that included $1 \mu \mathrm{l}$ of cDNA template $(\sim 5 \mathrm{ng}), 5 \mu \mathrm{l}$ of FastStart Universal SYBR-Green Master (Rox), $3.6 \mu \mathrm{l}$ of double-distilled water and $0.2 \mu \mathrm{l}$ of each pair of forward and

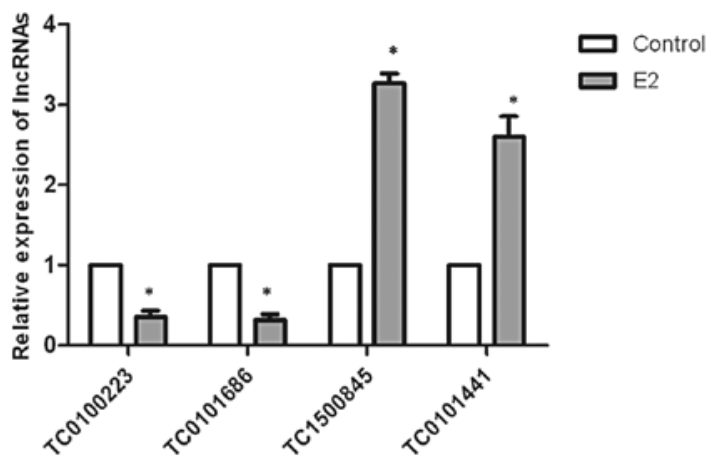

Figure 1. Relative expression of the four selected E2-regulated lncRNAs. The expression levels of four selected lncRNAs in the absence and presence of E2 in SKOV 3 cells. ${ }^{*} \mathrm{P}<0.05$ in comparison to E2- and vehicle-treated samples by qRT-PCR. Error bar denotes the standard deviation.

reverse primers (Table I; Sangon Biotech Co., Ltd., Shanghai, China). The PCR conditions included an initial denaturation step at $95^{\circ} \mathrm{C}$ for $10 \mathrm{~min}$ and 40 cycles of $95^{\circ} \mathrm{C}$ for $15 \mathrm{sec}$ and $60^{\circ} \mathrm{C}$ for $30 \mathrm{sec}$. All the experiments were performed in triplicate, and all the samples were normalized to GAPDH expression. The expression fold-changes were calculated using the $2^{-\Delta \Delta \mathrm{Ct}}$ method.

Statistical analysis. The data were processed using SPSS version 16.0 software (SPSS, Inc., Chicago, IL, USA). Comparison of continuous data was analyzed using the Student's t-test, whereas categorical data was analyzed using the Chi-square test and Fisher's exact test where appropriate (when the expected frequency was $<5$ ). OS curves were plotted according to the Kaplan-Meier method, with the log-rank test applied for comparison. Variables were used in multivariate analysis on the basis of the Cox proportional hazards model. A $\mathrm{P}$-value $<0.05$ was considered to indicate a statistically significant difference $(\mathrm{P}<0.05)$.

\section{Results}

Identification of E2-regulated lncRNAs in ER $\alpha+$ ovarian cancer cells

Identification of E2-regulated lncRNAs in SKOV3 cells. As our previous studies provided evidence that $\mathrm{E} 2$ regulated some protein-coding genes in ER $\alpha+$ ovarian cancer SKOV3 cells (5-7), we examined whether the expression of any lncRNAs is also regulated by E2 in SKOV3 cells. In the present study, SKOV3 cells were treated with $10^{-8}$ M E2 for $24 \mathrm{~h}$, and changes in the IncRNA expression profile were analyzed by performing a microarray. The microarray data indicated that 115 lncRNAs were significantly dysregulated following E2 treatment, including 51 upregulated and 64 downregulated lncRNAs (fold-change $\geq 1.5, \mathrm{P}<0.05$; data not shown). The top ten relative increased and decreased E2-regulated lncRNAs are listed in Table II.

To confirm the microarray findings, we examined the expression of four lncRNAs selected from the top ten relative increased and decreased E2-regulated 1ncRNAs using qRT-PCR. The results revealed that the expression levels of TC0100223 and TC0101686 were significantly downregulated by E2, whereas TC1500845 and TC0101441 were significantly 
Table II. The top ten relative increased and decreased E2-regulated lncRNAs in SKOV3 cells.

Annotations

\begin{tabular}{|c|c|c|c|c|c|c|}
\hline & & & & & & \\
\hline Probeset_id & Regulated & Fold-change (E2/control) & Seqname & Start & End & Strand \\
\hline TC0500815 & Upregulated & 3.419413635 & $\operatorname{chr} 5$ & 1043143 & 1050457 & - \\
\hline ТC0101441 & Upregulated & 3.275356271 & $\operatorname{chr} 1$ & 202377159 & 202378011 & + \\
\hline ТC0901107 & Upregulated & 3.269435942 & $\operatorname{chr} 9$ & 89871170 & 89871958 & - \\
\hline ТC0301101 & Upregulated & 3.258219523 & chr3 & 37825199 & 37878275 & - \\
\hline TC1900181 & Upregulated & 3.247328231 & $\operatorname{chr} 19$ & 10820078 & 10841404 & + \\
\hline TC1201706 & Upregulated & 3.232226492 & $\operatorname{chr} 12$ & 131237621 & 131240193 & - \\
\hline ТС0601086 & Upregulated & 3.186614845 & chr6 & 29802359 & 29824805 & - \\
\hline TC1500845 & Upregulated & 3.183351441 & $\operatorname{chr} 15$ & 38773376 & 38774597 & - \\
\hline ТC0300769 & Upregulated & 3.143316689 & $\operatorname{chr} 3$ & 169450147 & 169514658 & + \\
\hline TC0X00076 & Upregulated & 2.989272086 & $\operatorname{chrX}$ & 18821244 & 18823011 & + \\
\hline TC0501141 & Downregulated & 0.300418806 & $\operatorname{chr} 5$ & 90642594 & 90645975 & - \\
\hline TC1201365 & Downregulated & 0.301520979 & $\operatorname{chr} 12$ & 64556922 & 64561625 & - \\
\hline TC0101686 & Downregulated & 0.30390326 & $\operatorname{chr} 1$ & 244341256 & 244343791 & + \\
\hline TC1200811 & Downregulated & 0.31213872 & $\operatorname{chr} 12$ & 125146739 & 125152812 & + \\
\hline ТC0300928 & Downregulated & 0.318626574 & $\operatorname{chr} 3$ & 197148023 & 197150980 & + \\
\hline TC1900906 & Downregulated & 0.325966672 & $\operatorname{chr} 19$ & 61499140 & 61513631 & + \\
\hline TC0801241 & Downregulated & 0.328971081 & chr8 & 128289293 & 128300515 & - \\
\hline TC1400428 & Downregulated & 0.333717978 & $\operatorname{chr} 14$ & 88886369 & 88900796 & + \\
\hline TC0201596 & Downregulated & 0.335402449 & $\operatorname{chr} 2$ & 69870840 & 69879634 & - \\
\hline TC0100223 & Downregulated & 0.342972038 & $\operatorname{chr} 1$ & 21465570 & 21466331 & + \\
\hline
\end{tabular}
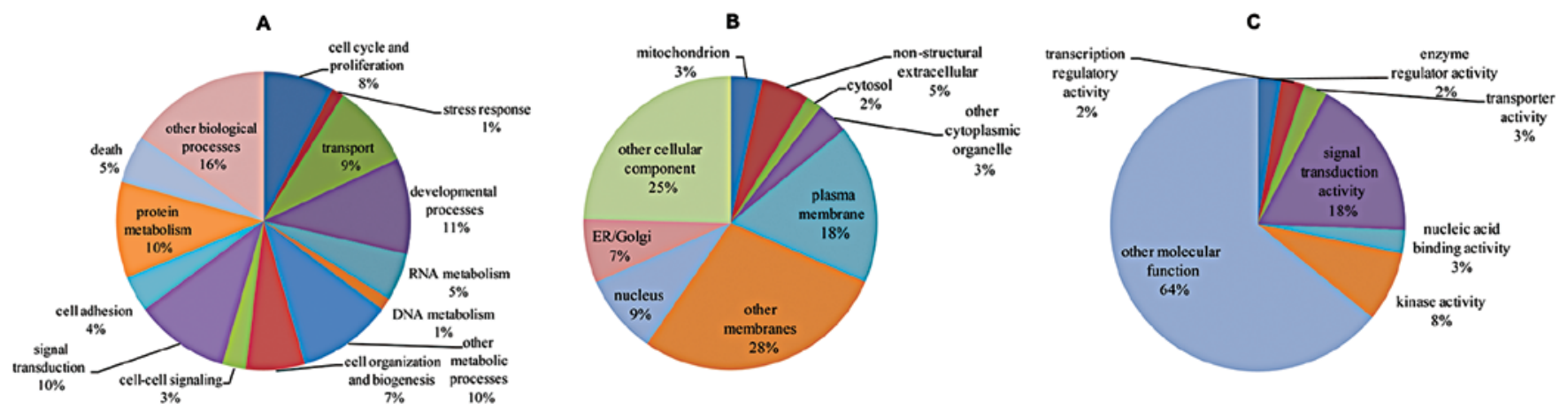

Figure 2. GO enrichment analysis of lncRNA targets. GO annotations of lncRNA targets categorized by (A) biological process, (B) cell component and (C) molecular function.

upregulated by E2 in SKOV3 cells, consistent with the microarray results (Fig. 1).

Putative targets of E2-regulated IncRNAs and their functional analysis. Based on the overlap between the targets predicted by bioinformatics and the differentially expressed mRNAs detected in the microarray, we constructed 55 E2-regulated lncRNA-target mRNA pairs (Table III). The GO (Fig. 2) and pathway (Table IV) analyses showed that a set of E2-regulated lncRNAs that mapped to the target mRNAs were correlated with several cellular processes and pathways known to be related to cancer progression, such as cell cycle and proliferation, developmental processes, cell adhesion, cell death,
MAPK signaling, Hedgehog signaling, Jak-STAT signaling and cancer pathways, suggesting their great potential to contribute to cancer progression.

Expression of several candidate lncRNAs in ER $\alpha+E O C$ tissues. In order to confirm the potential of some E2-regulated lncRNAs to contribute to cancer progression, we initially selected the four IncRNAs (TC0100223, TC0101686, TC1500845 and TC0101441) as candidates and tested their expression levels in EOC tissues. Considering the fact that $\mathrm{ER} \alpha$ is the main form expressed in malignant ovarian tumors and as ER $\alpha$ has been reported to promote poor prognosis in EOC patients $(28,29)$, we determined whether the expres- 
Table III. IncRNA-target mRNA pairs regulated by E2 in SKOV3 cells.

\begin{tabular}{|c|c|c|c|c|c|c|c|c|c|c|}
\hline \multicolumn{4}{|c|}{ Information of lncRNAs } & \multicolumn{3}{|c|}{ lncRNA-mRNA pairs } & \multicolumn{4}{|c|}{ Information of mRNAs } \\
\hline Start & End & Strand & Seqname & $\begin{array}{c}\text { lncRNA } \\
\text { Probeset_id }\end{array}$ & $\begin{array}{l}\text { mRNA } \\
\text { Symbol }\end{array}$ & Type & Start & End & Strand & Seqname \\
\hline 43821718 & 43827655 & + & $\operatorname{chr} 20$ & TC2000321 & $\mathrm{CD} 40$ & cis & 44180313 & 44366257 & + & $\operatorname{chr} 20$ \\
\hline 43821718 & 43827655 & + & chr20 & TC2000321 & UBE2C & cis & 43874662 & 43879003 & + & $\operatorname{chr} 20$ \\
\hline 63310787 & 63321606 & - & $\operatorname{chr} 19$ & TC1901868 & ZNF544 & cis & 63432092 & 63480673 & + & chr19 \\
\hline 63310787 & 63321606 & - & $\operatorname{chr} 19$ & TC1901868 & ZSCAN4 & cis & 62872115 & 62882317 & + & $\operatorname{chr} 19$ \\
\hline 63310787 & 63321606 & - & $\operatorname{chr} 19$ & TC1901868 & ZNF417 & cis & 63110053 & 63119796 & - & $\operatorname{chr} 19$ \\
\hline 63310787 & 63321606 & - & $\operatorname{chr} 19$ & TC1901868 & ZNF460 & cis & 62483670 & 62496635 & + & $\operatorname{chr} 19$ \\
\hline 61499140 & 61513631 & + & $\operatorname{chr} 19$ & TC1900906 & ZNF460 & cis & 62483670 & 62496635 & + & chr19 \\
\hline 10820078 & 10841404 & + & chr19 & TC1900181 & CDC37 & cis & 10362809 & 10375271 & - & chr19 \\
\hline 10820078 & 10841404 & + & chr19 & TC1900181 & QTRT1 & cis & 10673106 & 10805160 & + & chr19 \\
\hline 75925981 & 75926392 & + & $\operatorname{chr} 17$ & TC1700826 & KIAA1618 & cis & 75849262 & 75925295 & + & $\operatorname{chr} 17$ \\
\hline 29738362 & 29740074 & - & $\operatorname{chr} 16$ & TC1600989 & QPRT & cis & 29597859 & 29616810 & + & $\operatorname{chr} 16$ \\
\hline 70157455 & 70163889 & + & chr16 & TC1600575 & LOC652737 & cis & 69398791 & 69457733 & - & $\operatorname{chr} 16$ \\
\hline 38773376 & 38774597 & - & $\operatorname{chr} 15$ & TC1500845 & TYRO3 & cis & 39638524 & 39658826 & + & $\operatorname{chr} 15$ \\
\hline 38773376 & 38774597 & - & $\operatorname{chr} 15$ & TC1500845 & IVD & cis & 38484978 & 38515438 & + & $\operatorname{chr} 15$ \\
\hline 67751133 & 67757033 & + & $\operatorname{chr} 15$ & TC1500441 & PAQR5 & cis & 67378348 & 67486098 & + & $\operatorname{chr} 15$ \\
\hline 21379262 & 21379796 & + & $\operatorname{chr} 14$ & TC1400062 & ABHD4 & cis & 22136986 & 22151097 & + & $\operatorname{chr} 14$ \\
\hline 11599456 & 11603583 & + & $\operatorname{chr} 12$ & TC1200137 & TAS2R7 & cis & 10845399 & 10846493 & - & $\operatorname{chr} 12$ \\
\hline 7154170 & 7159091 & + & $\operatorname{chr} 12$ & TC1200076 & CDCA3 & cis & 6824224 & 6830686 & - & $\operatorname{chr} 12$ \\
\hline 7154170 & 7159091 & + & $\operatorname{chr} 12$ & TC1200076 & CLSTN3 & cis & 7174234 & 7202795 & + & $\operatorname{chr} 12$ \\
\hline 7154170 & 7159091 & + & $\operatorname{chr} 12$ & TC1200076 & ING4 & cis & 6629707 & 6642565 & - & $\operatorname{chr} 12$ \\
\hline 7154170 & 7159091 & + & $\operatorname{chr} 12$ & TC1200076 & PTPN6 & cis & 6926001 & 6940741 & + & $\operatorname{chr} 12$ \\
\hline 6966612 & 7033762 & + & $\operatorname{chr} 12$ & TC1200074 & CDCA3 & cis & 6824224 & 6830686 & - & $\operatorname{chr} 12$ \\
\hline 6966612 & 7033762 & + & $\operatorname{chr} 12$ & TC1200074 & CLSTN3 & cis & 7174234 & 7202795 & + & $\operatorname{chr} 12$ \\
\hline 6966612 & 7033762 & + & $\operatorname{chr} 12$ & TC1200074 & ING4 & cis & 6629707 & 6642565 & - & $\operatorname{chr} 12$ \\
\hline 6966612 & 7033762 & + & $\operatorname{chr} 12$ & TC1200074 & PTPN6 & cis & 6926001 & 6940741 & + & $\operatorname{chr} 12$ \\
\hline 5383589 & 5384883 & + & $\operatorname{chr} 12$ & TC1200045 & NTF3 & cis & 5473527 & 5474725 & + & $\operatorname{chr} 12$ \\
\hline 58457692 & 58582501 & - & $\operatorname{chr} 11$ & TC1101435 & STX3 & cis & 59279108 & 59326752 & + & chr11 \\
\hline 18821244 & 18823011 & + & $\operatorname{chrX}$ & TC0X00076 & GPR64 & cis & 18917348 & 19050676 & - & $\operatorname{chrX}$ \\
\hline 66450847 & 66456323 & - & $\operatorname{chr} 9$ & ТC0900981 & PIK3C2B & $\operatorname{trans}$ & $2.03 \mathrm{E}+08$ & 202730566 & - & $\operatorname{chr} 1$ \\
\hline 12264367 & 12270292 & + & chr8 & TC0800087 & DLC1 & cis & 12985243 & 13506486 & - & $\operatorname{chr} 8$ \\
\hline 12264367 & 12270292 & + & chr8 & TC0800087 & CTSB & cis & 11737442 & 11763147 & - & chr8 \\
\hline $1.28 \mathrm{E}+08$ & $1.28 \mathrm{E}+08$ & - & $\operatorname{chr} 7$ & TC0701620 & OPN1SW & cis & $1.28 \mathrm{E}+08$ & 128203087 & - & $\operatorname{chr} 7$ \\
\hline $1.28 \mathrm{E}+08$ & $1.28 \mathrm{E}+08$ & - & $\operatorname{chr} 7$ & TC0701620 & TSPAN33 & cis & $1.29 \mathrm{E}+08$ & 128595907 & + & $\operatorname{chr} 7$ \\
\hline $1.28 \mathrm{E}+08$ & $1.28 \mathrm{E}+08$ & - & $\operatorname{chr} 7$ & ТC0701620 & SMO & cis & $1.29 \mathrm{E}+08$ & 128640619 & + & $\operatorname{chr} 7$ \\
\hline $1.42 \mathrm{E}+08$ & $1.42 \mathrm{E}+08$ & + & $\operatorname{chr} 7$ & ТC0700794 & TAS2R5 & cis & $1.41 \mathrm{E}+08$ & 141137635 & + & $\operatorname{chr} 7$ \\
\hline $1.4 \mathrm{E}+08$ & $1.41 \mathrm{E}+08$ & + & $\operatorname{chr} 7$ & TC0700753 & TAS2R5 & cis & $1.41 \mathrm{E}+08$ & 141137635 & + & $\operatorname{chr} 7$ \\
\hline 29802359 & 29824805 & - & chr6 & TC0601086 & KIAA1949 & cis & 30752146 & 30763651 & - & chr6 \\
\hline $1.5 \mathrm{E}+08$ & $1.5 \mathrm{E}+08$ & - & $\operatorname{chr} 5$ & TC0501415 & TNIP1 & cis & $1.5 \mathrm{E}+08$ & 150446914 & - & $\operatorname{chr} 5$ \\
\hline $1.5 \mathrm{E}+08$ & $1.5 \mathrm{E}+08$ & - & $\operatorname{chr} 5$ & ТC0501415 & SLC36A1 & cis & $1.51 \mathrm{E}+08$ & 150852132 & + & $\operatorname{chr} 5$ \\
\hline $1.5 \mathrm{E}+08$ & $1.5 \mathrm{E}+08$ & - & chr5 & TC0501415 & ANXA6 & cis & $1.5 \mathrm{E}+08$ & 150517636 & - & $\operatorname{chr} 5$ \\
\hline $1.5 \mathrm{E}+08$ & $1.5 \mathrm{E}+08$ & - & $\operatorname{chr} 5$ & TC0501415 & CCDC69 & cis & $1.51 \mathrm{E}+08$ & 150583899 & - & $\operatorname{chr} 5$ \\
\hline 1043143 & 1050457 & - & $\operatorname{chr} 5$ & TC0500815 & LPCAT1 & cis & 1514544 & 1577092 & - & $\operatorname{chr} 5$ \\
\hline $1.15 \mathrm{E}+08$ & $1.15 \mathrm{E}+08$ & - & $\operatorname{chr} 4$ & ТC0401208 & ARSJ & cis & $1.15 \mathrm{E}+08$ & 115120306 & - & $\operatorname{chr} 4$ \\
\hline 37825199 & 37878275 & - & $\operatorname{chr} 3$ & TC0301101 & XYLB & cis & 38363244 & 38431471 & + & $\operatorname{chr} 3$ \\
\hline $1.35 \mathrm{E}+08$ & $1.35 \mathrm{E}+08$ & + & $\operatorname{chr} 3$ & TC0300635 & AMOTL2 & cis & $1.36 \mathrm{E}+08$ & 135576450 & - & $\operatorname{chr} 3$ \\
\hline $1.24 \mathrm{E}+08$ & $1.24 \mathrm{E}+08$ & + & $\operatorname{chr} 3$ & TC0300552 & STXBP5L & cis & $1.22 \mathrm{E}+08$ & 122621336 & + & $\operatorname{chr} 3$ \\
\hline
\end{tabular}


Table III. Continued.

\begin{tabular}{|c|c|c|c|c|c|c|c|c|c|c|}
\hline \multicolumn{4}{|c|}{ Information of lncRNAs } & \multicolumn{3}{|c|}{ lncRNA-mRNA pairs } & \multicolumn{4}{|c|}{ Information of mRNAs } \\
\hline Start & End & Strand & Seqname & $\begin{array}{c}\text { lncRNA } \\
\text { Probeset_id }\end{array}$ & $\begin{array}{l}\text { mRNA } \\
\text { Symbol }\end{array}$ & Type & Start & End & Strand & Seqname \\
\hline $1.14 \mathrm{E}+08$ & $1.14 \mathrm{E}+08$ & + & $\operatorname{chr} 2$ & TC0200609 & PAX8 & cis & $1.14 \mathrm{E}+08$ & 113752969 & - & $\operatorname{chr} 2$ \\
\hline $2.46 \mathrm{E}+08$ & $2.46 \mathrm{E}+08$ & - & chr1 & TC0103436 & ZNF670 & cis & $2.45 \mathrm{E}+08$ & 245308738 & - & chr1 \\
\hline $2.44 \mathrm{E}+08$ & $2.44 \mathrm{E}+08$ & + & $\operatorname{chr} 1$ & ТC0101686 & ZNF670 & cis & $2.45 \mathrm{E}+08$ & 245308738 & - & $\operatorname{chr} 1$ \\
\hline $2.02 \mathrm{E}+08$ & $2.02 \mathrm{E}+08$ & + & $\operatorname{chr} 1$ & TC0101441 & PIK3C2B & cis & $2.03 \mathrm{E}+08$ & 202730566 & - & $\operatorname{chr} 1$ \\
\hline $2.02 \mathrm{E}+08$ & $2.02 \mathrm{E}+08$ & + & $\operatorname{chr} 1$ & TC0101441 & ATP2B4 & cis & $2.02 \mathrm{E}+08$ & 201979832 & + & $\operatorname{chr} 1$ \\
\hline 53566493 & 53569511 & + & $\operatorname{chr} 1$ & TC0100566 & C1orf163 & cis & 52925096 & 52936964 & - & $\operatorname{chr} 1$ \\
\hline 53566493 & 53569511 & + & chr1 & TC0100566 & CC2D1B & cis & 52588855 & 52604453 & - & $\operatorname{chr} 1$ \\
\hline 21465570 & 21466331 & + & $\operatorname{chr} 1$ & TC0100223 & ECE1 & cis & 21417664 & 21544621 & - & $\operatorname{chr} 1$ \\
\hline 21465570 & 21466331 & + & chr1 & TC0100223 & RAP1GAP & cis & 21795301 & 21868437 & - & chr1 \\
\hline
\end{tabular}

Table IV. Target mRNA-related pathways in SKOV3 cells.

\begin{tabular}{|c|c|c|}
\hline Term & Count & Genes \\
\hline Pentose and glucuronate interconversions & 1 & XYLB \\
\hline Valine, leucine and isoleucine degradation & 1 & IVD \\
\hline Inositol phosphate metabolism & 1 & $\mathrm{PIK} 3 \mathrm{C} 2 \mathrm{~B}$ \\
\hline Nicotinate and nicotinamide metabolism & 1 & QPRT \\
\hline Metabolic pathways & 4 & IVD, QPRT, XYLB, PIK3C2B \\
\hline MAPK signaling pathway & 1 & NTF3 \\
\hline Phosphatidylinositol signaling system & 1 & $\mathrm{PIK} 3 \mathrm{C} 2 \mathrm{~B}$ \\
\hline Ubiquitin mediated proteolysis & 1 & UBE2C \\
\hline SNARE interactions in vesicular transport & 1 & STX3 \\
\hline Hedgehog signaling pathway & 1 & SMO \\
\hline Adherens junction & 1 & PTPN6 \\
\hline Jak-STAT signaling pathway & 1 & PTPN6 \\
\hline Natural killer cell mediated cytotoxicity & 1 & PTPN6 \\
\hline $\mathrm{T}$ cell receptor signaling pathway & 1 & PTPN6 \\
\hline B cell receptor signaling pathway & 1 & PTPN6 \\
\hline Neurotrophin signaling pathway & 1 & NTF3 \\
\hline Pathways in cancer & 1 & SMO \\
\hline Basal cell carcinoma & 1 & SMO \\
\hline
\end{tabular}

sion of in vitro E2-regulated lncRNAs, detected in ER $\alpha+$ ovarian cancer cells, could discriminate between ER $\alpha+$ and ER $\alpha$ - EOC tissues. Based on the qRT-PCR assay, we found that ER $\alpha+$ tissues had lower expression of TC0100223 and TC0101686 and higher expression of TC0101441 (Fig. 3, $\mathrm{ER} \alpha+, \mathrm{n}=64$ vs. $\mathrm{ER} \alpha-, \mathrm{n}=31, \mathrm{P}<0.01$; Fig. 3A shows the representative immunohistochemistry results of ER $\alpha$ expression in EOC tissues). In contrast, TC1500845 was not differentially expressed between ER $\alpha+$ and ER $\alpha$ - EOC tissues. These results may be suggestive of the potential clinical significance of TC0100223, TC0101686 and TC0101441 in $\mathrm{ER} \alpha+\mathrm{EOC}$.
Association of IncRNA expression with clinicopathological characteristics in ER $\alpha+E O C$. According to the median value which was used as the cut-off (30), specific IncRNA expression in ER $\alpha+$ EOC tissues, equal or more than median value was defined as high lncRNA group, and less than median value was defined as low lncRNA group. As shown in Table V, low-expression of TC0100223 and TC0101686 and high-expression of TC0101441 were closely related to ER $\alpha+$ EOC tissues with advanced FIGO stage and/or high histological grade $(\mathrm{P}<0.05)$, suggesting that aberrant expression of the three candidate lncRNAs is associated with a more malignant ovarian cancer phenotype. 
Table V. Association of lncRNA expression with clinicopathological variables in ER $\alpha$-positive EOC patients.

\begin{tabular}{|c|c|c|c|c|c|c|c|}
\hline \multirow[b]{2}{*}{ Variables } & \multirow[b]{2}{*}{ Cases $(\mathrm{N})$} & \multicolumn{2}{|c|}{$\begin{array}{l}\text { High TC0101441 } \\
\text { expression }\end{array}$} & \multicolumn{2}{|c|}{$\begin{array}{c}\text { Low TC0100223 } \\
\text { expression }\end{array}$} & \multicolumn{2}{|c|}{$\begin{array}{c}\text { Low TC0101686 } \\
\text { expression }\end{array}$} \\
\hline & & $\mathrm{n}(\%)$ & P-value & n $(\%)$ & P-value & $\mathrm{n}(\%)$ & P-value \\
\hline \multicolumn{8}{|c|}{ Age (years) } \\
\hline$<50$ & 25 & $14(56.0)$ & 0.442 & $10(40.0)$ & 0.2 & $15(60.0)$ & 0.2 \\
\hline$\geq 50$ & 39 & $18(46.2)$ & & $22(56.4)$ & & $17(43.6)$ & \\
\hline \multicolumn{8}{|c|}{ Histological subtype } \\
\hline Serous & 49 & $23(46.9)$ & 0.376 & $24(49.0)$ & 0.768 & $22(44.9)$ & 0.14 \\
\hline Other & 15 & $9(60.0)$ & & $8(53.3)$ & & $10(66.7)$ & \\
\hline \multicolumn{8}{|c|}{ FIGO stage } \\
\hline $\mathrm{I}-\mathrm{II}$ & 24 & $5(20.8)$ & $<0.001$ & $8(33.3)$ & 0.039 & 7 (29.2) & 0.01 \\
\hline III-IV & 40 & $27(67.5)$ & & $24(60.0)$ & & $25(62.5)$ & \\
\hline \multicolumn{8}{|c|}{ Histological grade } \\
\hline G1-G2 & 27 & $6(22.2)$ & $<0.001$ & $9(33.3)$ & 0.023 & $11(40.7)$ & 0.206 \\
\hline G3 & 37 & $26(70.3)$ & & $23(62.2)$ & & $21(56.8)$ & \\
\hline \multicolumn{8}{|l|}{ Ascites } \\
\hline$>100$ & 20 & $11(55.0)$ & 0.59 & $7(35.0)$ & 0.106 & $12(60.0)$ & 0.281 \\
\hline$\geq 100$ & 44 & $21(47.7)$ & & $25(56.8)$ & & $20(45.5)$ & \\
\hline
\end{tabular}

\section{A}

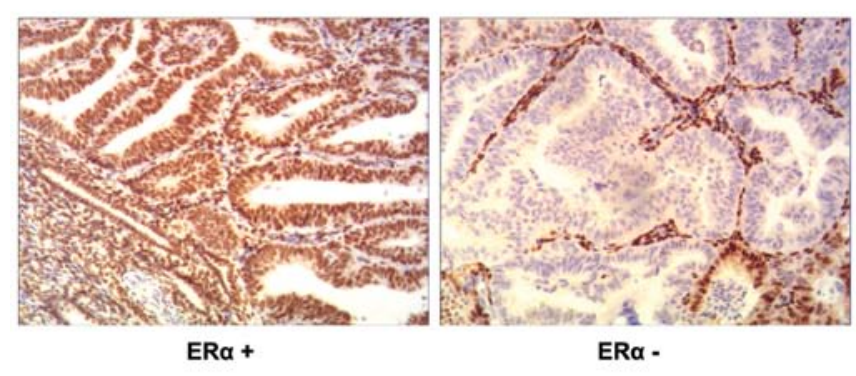

B
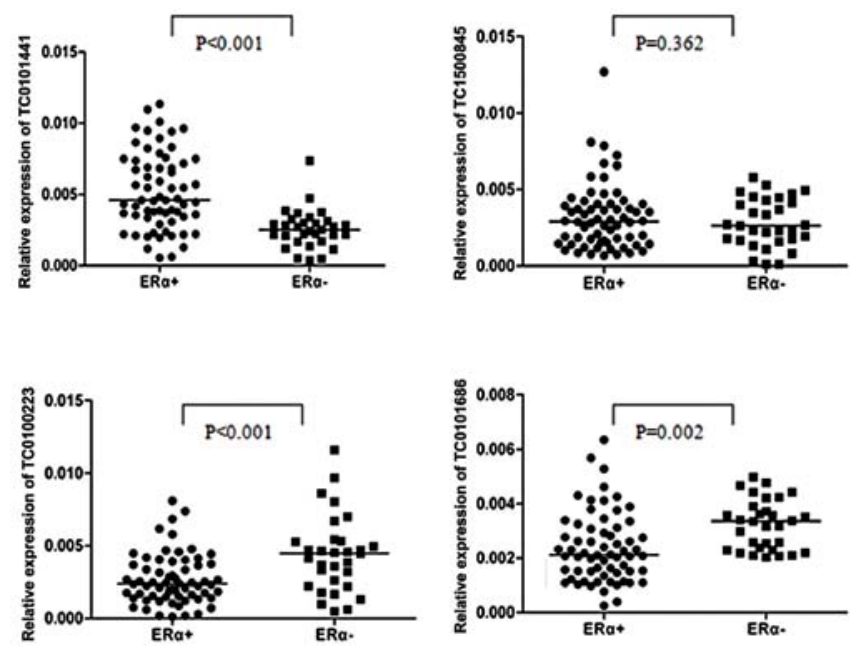

Figure 3. IncRNA expression in EOC tissues (ER $\alpha+$ vs. ER $\alpha-)$. (A) Representative immunohistochemistry results of ER $\alpha$ expression in EOC tissues; original magnification, x200. (B) Relative expression levels of TC0101441, TC1500845, TC0100223 and TC0101686 in EOC tissues.



Figure 4. Survival curves of ER $\alpha+$ EOC patients according to TC0101441 expression. Patients with high TC0101441 expression showed significantly poorer prognosis than those with low TC0101441 expression $(\mathrm{P}<0.01, \log$-rank test).

Association of IncRNA expression with prognosis of ER $\alpha+$ EOC patients. We investigated whether the expression of TC0100223, TC0101686 and TC0101441 correlated with the postoperative survival of ER $\alpha+$ EOC patients. Among the 64 $\mathrm{ER} \alpha+\mathrm{EOC}$ patients, 38 died during follow-up. In univariate analysis, OS was associated with the FIGO stage, histological grade and expression of TC0100223 and TC0101441 $(\mathrm{P}<0.05$; Table VI). Multivariate analysis further confirmed that high TC0101441 expression, advanced FIGO stage and high histological grade were independent factors for evaluation of OS in ER $\alpha+$ EOC patients $(\mathrm{P}<0.05$, Table VI; Fig. 4 shows the OS curves according to TC0101441 expression). Thus, it was concluded that of the three candidate lncRNAs, TC0101441 could be used as an independent prognostic factor for OS of $\mathrm{ER} \alpha+\mathrm{EOC}$ patients. 
Table VI. Univariate analysis for overall survival in ER $\alpha$-positive EOC patients.

\begin{tabular}{|c|c|c|c|c|c|c|c|c|}
\hline \multirow[b]{3}{*}{ Variables } & \multicolumn{2}{|c|}{ Univariate analysis } & \multicolumn{6}{|c|}{ Multivariate analysis } \\
\hline & \multirow{2}{*}{$\frac{\text { Overall survival }}{\text { Mean } \pm \text { SE (months) }}$} & \multirow[b]{2}{*}{ P-value } & \multicolumn{6}{|c|}{ Overall survival } \\
\hline & & & $\beta$ & SE & Wald & P-value & $\operatorname{Exp}(\beta)$ & $95 \% \mathrm{CI}$ \\
\hline \multicolumn{9}{|c|}{ Age (years) } \\
\hline$<50$ & $45.52 \pm 4.21$ & 0.939 & - & - & - & - & - & - \\
\hline$\geq 50$ & $47.49 \pm 3.98$ & & - & - & - & - & - & - \\
\hline \multicolumn{9}{|c|}{ Histological subtype } \\
\hline Serous & $45.99 \pm 3.53$ & 0.496 & - & - & - & - & - & - \\
\hline Other & $52.30 \pm 5.71$ & & - & - & - & - & - & - \\
\hline \multicolumn{9}{|c|}{ FIGO stage } \\
\hline I-II & $69.63 \pm 2.42$ & & - & - & - & - & - & - \\
\hline III-IV & $33.03 \pm 2.73$ & $<0.001$ & 2.305 & 0.635 & 13.168 & $<0.001$ & 10.022 & $2.886-34.803$ \\
\hline \multicolumn{9}{|c|}{ Histological grade } \\
\hline $\mathrm{G} 1-\mathrm{G} 2$ & $64.81 \pm 2.97$ & & - & - & - & - & - & - \\
\hline G3 & $32.62 \pm 2.90$ & $<0.001$ & 0.991 & 0.481 & 4.238 & 0.04 & 2.693 & $1.049-6.915$ \\
\hline \multicolumn{9}{|l|}{ Ascites } \\
\hline$<100$ & $44.71 \pm 5.95$ & 0.77 & - & - & - & - & - & - \\
\hline$\geq 100$ & $48.67 \pm 3.48$ & & - & - & - & - & - & - \\
\hline \multicolumn{9}{|c|}{ TC0101686 expression } \\
\hline Low & $44.45 \pm 4.23$ & 0.325 & - & - & - & - & - & - \\
\hline High & $50.32 \pm 4.28$ & & - & - & - & - & - & - \\
\hline \multicolumn{9}{|c|}{ TC0100223 expression } \\
\hline Low & $37.48 \pm 3.48$ & 0.018 & - & - & - & - & - & - \\
\hline High & $54.40 \pm 3.99$ & & 0.019 & 0.352 & 0.003 & 0.957 & 0.981 & $0.492-1.956$ \\
\hline \multicolumn{9}{|c|}{ TC0101441 expression } \\
\hline Low & $60.88 \pm 3.48$ & & - & - & - & - & - & - \\
\hline High & $32.16 \pm 3.04$ & $<0.001$ & 0.817 & 0.402 & 4.122 & 0.042 & 2.263 & $1.029-4.979$ \\
\hline
\end{tabular}

$\beta$, regression coefficient; SE, standard error; CI, confidence interval.

\section{Discussion}

In the present study, we identified a series of differentially expressed E2-regulated lncRNAs in ER $\alpha+$ ovarian cancer SKOV3 cells using a microarray. Bioinformatics functional analyses indicated that a fraction of these lncRNAs had the potential to contribute to cancer progression. Furthermore, in order to confirm that some E2-regulated lncRNAs are related to the development of ER $\alpha+\mathrm{EOC}$, we tested the expression of several candidate lncRNAs in EOC tissues. The results showed that some candidate lncRNAs were aberrantly expressed in $\mathrm{ER} \alpha+$ compared to ER $\alpha$ - EOC tissues, and their differential expression was associated with certain clinicopathological variables and poor prognosis of ER $\alpha+E O C$. To the best of our knowledge, this is the first study to report E2-regulated lncRNAs in $\mathrm{ER} \alpha+\mathrm{EOC}$, the results of which may provide insight into the estrogenic effects on EOC progression and the design of new target therapies based on a perspective of IncRNA.

It is known that the ovary is a main source and target tissue of E2 in women. The action of E2 on ovarian tissue is believed to be mediated by the two ERs, ER $\alpha$ and $\operatorname{ER} \beta$. ER $\beta$ is highly expressed in normal epithelial ovarian cells and benign tumors, whereas $\mathrm{ER} \alpha$ is expressed to a much greater extent in malignant ovarian tumors (28). Several studies thus far have revealed the contributions to EOC progression by multiple E2/ER $\alpha$-regulated target protein-coding genes, such as cyclin D1 and c-myc (which are involved in cellular growth control) and fibulin-1 and cathepsin-D (which are involved in cellular motility and invasion) $(10,11)$.Our previous studies also showed that E2 promoted metastasis and invasion in ER $\alpha+$ ovarian cancer SKOV3 cells by regulating HIF-1, nm23-H1, E-cadherin and MMP-2 (5-7). Despite these protein-coding genes, however, the exact effects of E2 on EOC development remain unclear. In the present study, we identified 115 E2-regulated lncRNAs in ER $\alpha+$ SKOV3 cells using a microarray, and subsequent bioinformatics analyses indicated that a subset of these lncRNAs had the potential to contribute to cancer progression. These findings may extend our current knowledge regarding E2 regulation of protein-coding genes in EOC progression to include lncRNAs. 
lncRNAs, initially argued to be spurious transcriptional noise, are emerging as new regulators in the cancer paradigm. Misregulation of lncRNAs has been documented in many types of human cancer. For example, DDC and PCGEM are overexpressed in prostate cancer compared to normal prostate tissue, implicating their roles in tumorigenesis (31,32). Increased expression of MALAT1 indicates a poorer clinical outcome of lung cancer patients (15). HOTAIR is overexpressed in primary breast tumors and metastases, and elevated HOTAIR expression is an indispensable predictor of eventual metastasis and mortality (14). Inspired by these lines of evidence of IncRNA roles in cancer biology, we hypothesized that certain E2-regulated lncRNAs detected in SKOV3 cells in the current study may also have the potential to contribute to EOC progression. To address this hypothesis, we initially tested two upregulated (TC1500845 and TC0101441) and two downregulated lncRNAs (TC0100223 and TC0101686) in EOC tissues, the results of which showed a significant correlation of overexpressed TC0101441 and lowexpressed TC0100223 and TC0101686 with ER $\alpha+$ compared to ER $\alpha$ - EOC. Moreover, low-expression of TC0100223 and TC0101686 and overexpression of TC0101441 were found to be related to ER $\alpha+$ EOC tissues with advanced FIGO stage and/or high histological grade. Most importantly, multivariate survival analysis revealed that TC0101441 was an independent prognostic factor for overall survival. Taken together, our findings suggest that the aberrant expression of certain E2-regulated IncRNAs is associated with malignant cancer phenotypes and poor clinical outcome of ER $\alpha+$ EOC patients. Hence, these results may also lead us to consider that E2-regulated lncRNAs can be used as candidate biomarkers for EOC prognosis and therapy. Clearly, further studies are required to elucidate the roles and mechanisms by which these lncRNAs promote EOC development in detail.

In conclusion, the present study provided the first evidence that E2 can modulate a panel of lncRNAs in ER $\alpha+$ EOC cells. Some aberrantly expressed lncRNAs, including TC0100223, TC0101686 and TC0101441, are correlated with the advanced cancer phenotypes. Of note, TC0101441 was an independent factor for poor prognosis of ER $\alpha+$ EOC patients. Collectively, encouraged by the involvement of these E2-regulated lncRNAs in ER $\alpha+$ EOC progression, our data highlight the utility of considering lncRNA expression in the future understanding of estrogenic effects on EOC progression and in the design of new target therapies.

\section{Acknowledgements}

The authors are grateful to the department managers who provided the microarray services at the National Engineering Center for Biochip at the Shanghai Biotechnology Corporation. The project described was supported by a Grant Number 81370689 from the National Natural Science Foundation of China.

\section{References}

1. Jemal A, Bray F, Center MM, Ferlay J, Ward E and Forman D: Global cancer statistics. CA Cancer J Clin 61: 69-90, 2011.

2. Siegel R, Naishadham D and Jemal A: Cancer statistics, 2012. CA Cancer J Clin 62: 10-29, 2012.
3. Tsilidis KK, Allen NE, Key TJ, Dossus L, Kaaks R, Bakken K, Lund E, Fournier A, Dahm CC, Overvad K, Hansen L, Tjønneland A, Rinaldi S, Romieu I, Boutron-Ruault MC, Clavel-Chapelon F, Lukanova A, Boeing $H$, Schütze $M$, Benetou V, Palli D, Berrino F, Galasso R, Tumino R, Sacerdote C, Bueno-de-Mesquita HB, van Duijnhoven FJ, Braem MG, Onland-Moret NC, Gram IT, Rodríguez L, Duell EJ, Sánchez MJ, Huerta JM, Ardanaz E, Amiano P, Khaw KT, Wareham N and Riboli E: Menopausal hormone therapy and risk of ovarian cancer in the European prospective investigation into cancer and nutrition. Cancer Causes Control 22: 1075-1084, 2011.

4. Park SH, Cheung LW, Wong AS and Leung PC: Estrogen regulates snail and slug in the down-regulation of E-cadherin and induces metastatic potential of ovarian cancer cells through estrogen receptor alpha. Mol Endocrinol 22: 2085-2098, 2008.

5. Hua K, Din J, Cao Q, Feng W, Zhang Y, Yao L, Huang Y, Zhao Y and Feng Y: Estrogen and progestin regulate HIF-1 $\alpha$ expression in ovarian cancer cell lines via the activation of Akt signaling transduction pathway. Oncol Rep 21: 893-898, 2009.

6. Hua K, Feng W, Cao Q, Zhou X, Lu X and Feng Y: Estrogen and progestin regulate metastasis through the PI3K/AKT pathway in human ovarian cancer. Int J Oncol 33: 959-967, 2008.

7. Ding JX, Feng YJ, Yao LQ, Yu M, Jin HY and Yin LH: The reinforcement of invasion in epithelial ovarian cancer cells by $17 \beta$-estradiol is associated with upregulation of Snail. Gynecol Oncol 103: 623-630, 2006.

8. Spillman MA, Manning NG, Dye WW, Sartorius CA, Post MD, Harrell JC, Jacobsen BM and Horwitz KB: Tissue-specific pathways for estrogen regulation of ovarian cancer growth and metastasis. Cancer Res 70: 8927-8936, 2010.

9. Laviolette LA, Garson K, Macdonald EA, Senterman MK, Courville K, Crane CA and Vanderhyden BC: 17ß-estradiol accelerates tumor onset and decreases survival in a transgenic mouse model of ovarian cancer. Endocrinology 151: 929-938, 2010.

10. Twal WO, Czirok A, Hegedus B, Knaak C, Chintalapudi MR, Okagawa H, Sugi Y and Argraves WS: Fibulin-1 suppression of fibronectin regulated cell adhesion and motility. J Cell Sci 114: 4587-4598, 2001

11. Rochefort H, Chalbos D, Cunat S, Lucas A, Platet N and Garcia M: Estrogen regulated proteases and antiproteases in ovarian and breast cancer cells. J Steroid Biochem Mol Biol 76: 119-124, 2001.

12. Wilusz JE, Sunwoo H and Spector DL: Long noncoding RNAs: functional surprises from the RNA world. Genes Dev 23: 1494-1504, 2009

13. Nagano $T$ and Fraser P: No-nonsense functions for long noncoding RNAs. Cell 145: 178-181, 2011.

14. Gupta RA, Shah N, Wang KC, Kim J, Horlings HM, Wong DJ, Tsai MC, Hung T, Argani P, Rinn JL, Wang Y, Brzoska P, Kong B, Li R, West RB, van de Vijver MJ, Sukumar S and Chang HY: Long non-coding RNA HOTAIR reprograms chromatin state to promote cancer metastasis. Nature 464: 1071-1076, 2010.

15. Tano K, Mizuno R, Okada T, Rakwal R, Shibato J, Masuo Y, Ijiri K and Akimitsu N: MALAT-1 enhances cell motility of lung adenocarcinoma cells by influencing the expression of motilityrelated genes. FEBS Lett 584: 4575-4580, 2010.

16. Matouk IJ, DeGroot N, Mezan S, Ayesh S, Abu-lail R, Hochberg A and Galun E: The H19 non-coding RNA is essential for human tumor growth. PLoS One 2: e845, 2007.

17. Du Y, Kong G, You X, Zhang S, Zhang T, Gao Y, Ye L and Zhang X: Elevation of highly up-regulated in liver cancer (HULC) by hepatitis B virus $\mathrm{X}$ protein promotes hepatoma cell proliferation via down-regulating p18. J Biol Chem 287: 26302-26311, 2012.

18. Huarte M, Guttman M, Feldser D, Garber M, Koziol MJ, Kenzelmann-Broz D, Khalil AM, Zuk O, Amit I, Rabani M, Attardi LD, Regev A, Lander ES, Jacks T and Rinn JL: A large intergenic noncoding RNA induced by $\mathrm{p} 53$ mediates global gene repression in the p53 response. Cell 142: 409-419, 2010.

19. Zhou Y, Zhang X and Klibanski A: MEG3 noncoding RNA: a tumor suppressor. J Mol Endocrinol 48: R45-R53, 2012.

20. Silva JM, Boczek NJ, Berres MW, Ma X and Smith DI: LSINCT5 is over expressed in breast and ovarian cancer and affects cellular proliferation. RNA Biol 8: 496-505, 2011.

21. Rangel LB, Sherman-Baust CA, Wernyj RP, Schwartz DR, Cho KR and Morin PJ: Characterization of novel human ovarian cancer-specific transcripts (HOSTs) identified by serial analysis of gene expression. Oncogene 22: 7225-7232, 2003. 
22. $\mathrm{Xu}$ W, Seok J, Mindrinos MN, Schweitzer AC, Jiang H, Wilhelmy J, Clark TA, Kapur K, Xing Y, Faham M, Storey JD, Moldawer LL, Maier RV, Tompkins RG, Wong WH, Davis RW and Xiao W: Human transcriptome array for high-throughput clinical studies. Proc Natl Acad Sci USA 108: 3707-3712, 2011.

23. Jia H, Osak M, Bogu GK, Stanton LW, Johnson R and Lipovich L: Genome-wide computational identification and manual annotation of human long noncoding RNA genes. RNA 16: 1478-1487, 2010.

24. Tafer H and Hofacker IL: RNAplex: a fast tool for RNA-RNA interaction search. Bioinformatics 24: 2657-2663, 2008.

25. Dennis G Jr, Sherman BT, Hosack DA, Yang J, Gao W, Lane HC and Lempicki RA: DAVID: Database for Annotation, Visualization, and Integrated Discovery. Genome Biol 4: P3, 2003.

26. Zhang C, Han L, Zhang A, Yang W, Zhou X, Pu P, Du Y, Zeng H and Kang C: Global changes of mRNA expression reveals an increased activity of the interferon-induced signal transducer and activator of transcription (STAT) pathway by repression of miR-221/222 in glioblastoma U251 cells. Int J Oncol 36: $1503-1512,2010$.

27. Yang XY, Xi MR, Yang KX and Yu H: Prognostic value of estrogen receptor and progesterone receptor status in young Chinese ovarian carcinoma patients. Gynecol Oncol 113: 99-104, 2009.

28. Pujol P, Rey JM, Nirde P, Roger P, Gastaldi M, Laffargue F, Rochefort $\mathrm{H}$ and Maudelonde T: Differential expression of estrogen receptor- $\alpha$ and $-\beta$ messenger RNAs as a potential marker of ovarian carcinogenesis. Cancer Res 58: 5367-5373, 1998.
29. Darb-Esfahani S, Wirtz RM, Sinn BV, Budczies J, Noske A, Weichert W, Faggad A, Scharff S, Sehouli J, Oskay-Ozcelik G, Zamagni C, De Iaco P, Martoni A, Dietel M and Denkert C: Estrogen receptor $1 \mathrm{mRNA}$ is a prognostic factor in ovarian carcinoma: determination by kinetic PCR in formalin-fixed paraffin-embedded tissue. Endocr Relat Cancer 16: 1229-1239, 2009.

30. Yuan SX, Yang F, Yang Y, Tao QF, Zhang J, Huang G, Yang Y Wang RY, Yang S, Huo XS, Zhang L, Wang F, Sun SH and Zhou WP: Long noncoding RNA associated with microvascular invasion in hepatocellular carcinoma promotes angiogenesis and serves as a predictor for hepatocellular carcinoma patients poor recurrence-free survival after hepatectomy. Hepatology 56: 2231-2241, 2012.

31. Bussemakers MJ, van Bokhoven A, Verhaegh GW, Smit FP, Karthaus HF, Schalken JA, Debruyne FM, Ru N and Isaacs WB: DD3: a new prostate-specific gene, highly overexpressed in prostate cancer. Cancer Res 9: 5975-5979, 1999.

32. Srikantan V, Zou Z, Petrovics G, Xu L, Augustus M, Davis L, Livezey JR, Connell T, Sesterhenn IA, Yoshino K, Buzard GS, Mostofi FK, McLeod DG, Moul JW and Srivastava S: PCGEM1, a prostate-specific gene, is overexpressed in prostate cancer. Proc Natl Acad Sci USA 97: 12216-12221, 2000. 